\title{
OBSERVATORIO
}

\section{LIDERAZGO Y EXCELENCIA DE LA CIENCIA ESPAÑOLA}

\author{
Félix De-Moya-Anegón
}

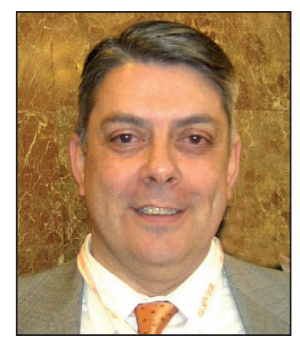

Félix De-Moya-Anegón, doctor en historia por la Universidad de Granada, es profesor de investigación en el Instituto de Políticas y Bienes Públicos del Consejo Superior de Investigaciones Científicas en Madrid. Es el investigador principal de la unidad asociada Grupo SCImago que ha llevado a cabo los proyectos de I+D Scimago Journal \& Country Rank (SJR) y Scimago Institutions Rankings (SIR), Atlas de la Ciencia, etc. Su última publicación web es el Ranking Iberoamericano SIR 2010.

Centro de Ciencias Humanas y Sociales (CCHS), CSIC Albasanz, 26-28, 28037 Madrid, España felix.moya@scimago.es

\section{Resumen}

España ocupa el lugar 10 en producción científica, pero en impacto se sitúa en el 22. Se analiza la distribución de la producción científica entre las 17 comunidades autónomas españolas. Madrid y Catalunya concentran el $49 \%$ de la producción total y el $58 \%$ de la excelente -la que está en el $10 \%$ mundial más citada-.

\section{Palabras clave}

Producción científica, España, Comunidades autónomas, Distribución, Comparación regional.

Title: Leadership and excellence of Spanish science

\section{Abstract}

Although Spain is ranked 10th in scientific production, it is 22 nd in impact. The distribution of scientific production across the 17 Spanish regions is analyzed. Madrid and Catalonia account for $49 \%$ of total production and for $58 \%$ of excellence as reflected in impact, defined as publications within the $10 \%$ most cited worldwide.

\section{Keywords}

Scientific output, Scientific production, Spain, Autonomous communities, Distribution, Regional comparison.

De-Moya-Anegón, Félix. "Liderazgo y excelencia de la ciencia española". El profesional de la información, 2012, marzoabril, v. 21, n. 2, pp. 125-128.

\section{Introducción}

No es ninguna novedad la alta producción científica de los investigadores españoles y de las instituciones, en su mayoría públicas, en las que trabajan. A esta situación se ha llegado, como es bien sabido, a través de un proceso de crecimiento muy acelerado de resultados de investigación publicados que nos colocó hace algunos años entre los diez primeros países del mundo en producción científica registrada internacionalmente. Tampoco resulta novedoso hoy decir que ese crecimiento casi vertiginoso de nuestras publicaciones de investigación no ha venido acompañado en todos los casos de un crecimiento similar de la calidad de esas mismas publicaciones. De hecho, si hacemos el ejercicio simplista de comparar la posición mundial de España en cuanto a producción científica (10) con su posición en cuanto al impacto promedio de esa producción -o sea, citas recibidas- (22), la conclusión parece clara. Producimos mucho pero su calidad media no es muy alta.

Sin embargo, una mirada más detenida a los resultados de investigación de los últimos años nos permitirá constatar la coexistencia de varios mundos en los resultados de investigación en los que participan los investigadores radicados en nuestro país. Como sucede también en otros países, en nuestro sistema de ciencia hay una masa importante de investigadores cuyos resultados apenas consiguen repercusión alguna en sus respetivas comunidades científicas y mucho menos en la sociedad. Por el contrario, un grupo más reducido de ellos alcanzan resultados de gran relevancia científica y también social.

Este fenómeno se refleja mal en los promedios a los que con insistencia se alude para poner de manifiesto nuestra posición en el mundo, especialmente desde el punto de vista 


\section{Posición de España}

En producción científica

En producción excelente

En impacto

Ritmo de crecimiento anual

Lo importante no es producir mucho, sino producir aquello que realmente hace avanzar el conocimiento, aunque por otra parte, sólo si se produce mucho se tiene masa crítica para que algo sea realmente valioso

cualitativo. Hoy lo importante en los procesos de generación de conocimiento científico no es producir mucho, sino producir aquello que realmente hace avanzar el conocimiento en los diferentes ámbitos de la ciencia, porque sabemos que es sólo una pequeña parte del conocimiento científico generado el que finalmente contribuirá a resolver los problemas que importan y preocupan en las sociedades avanzadas. Es verdad que la generación de este tipo de conocimiento hoy día es entendida y analizada como un fenómeno de masa crítica. De tal suerte que sólo si produces mucho tendrás la posibilidad de producir algo realmente útil y/o valioso. Pero también es cierto que una distribución homogénea de recursos entre los diferentes actores en un sistema de ciencia será el fruto de políticas que enfatizan la cantidad más que la calidad. Y, por el contrario, una política basada en la distribución discriminada de los recursos en favor de los mejores parece enfatizar la calidad de los resultados de investigación frente a la cantidad. Por estas y otras razones es preciso ir más allá de la visión que nos ofrecen los promedios y dar una mirada a la eficiencia de nuestro sistema de ciencia desde el punto de vista de nuestra contribución al avance real del conocimiento científico global.

\section{Distribución geográfica desigual}

El dominio científico español ha mantenido en los primeros años de este siglo XXI un ritmo de crecimiento de sus resultados de investigación con visibilidad internacional en los que participan sus investigadores de un $11 \%$ anual. Esta tasa de crecimiento es más alta que la de todos los grandes productores de ciencia en el mundo. Sólo algunos de los llamados países BRIC (Brasil, Rusia, India y China), entre los grandes productores, han crecido más aceleradamente que España en esos mismos años. Por otro lado, el grado de participación de nuestros resulta-

dos de ciencia entre el $10 \%$ de los trabajos científicos más citados del mundo por campo (producción de excelencia), casi no se ha movido del $11 \%$ de nuestra producción total en el mismo período.

Estos indicadores ponen de manifiesto que España no sólo está evolucionando de forma positiva en cuanto a su capacidad productiva en ciencia, sino que mantiene un nivel aceptable en cuanto a su potencial para generar conocimiento científico de alto nivel que en definitiva es el mejor síntoma de la capacidad de nuestro sistema de ciencia para transformar ese conocimiento en innovación. Es verdad que nuestra producción de excelencia nos sitúa en una muy buena posición en el mundo (11) y que su crecimiento $(81 \%)$ ha sido muy similar al de nuestro crecimiento bruto en el período 2003-2009. Pero también es cierto que el análisis de la aportación por CCAA e instituciones de la excelencia científica española revela desigualdades que ponen de manifiesto que nuestro sistema de generación de conocimiento científico, como otros en el mundo, se ha desarrollado distribuyendo desigualmente las mejores capacidades científicas.

\section{Una política basada en la distribución discriminada de los recursos en favor de los mejores parece enfatizar la calidad de los resultados de investigación frente a la cantidad}

En nuestro caso se concentra en instituciones ubicadas en Madrid y Catalunya principalmente, aunque en otras regiones se hacen esfuerzos significativos por no quedar descolgados del tren de la excelencia. En términos de producción científica bruta Madrid y Catalunya representan alrededor del $49 \%$ de la producción científica española, mientras que han acumulado a lo largo del mismo período el 58\% de la

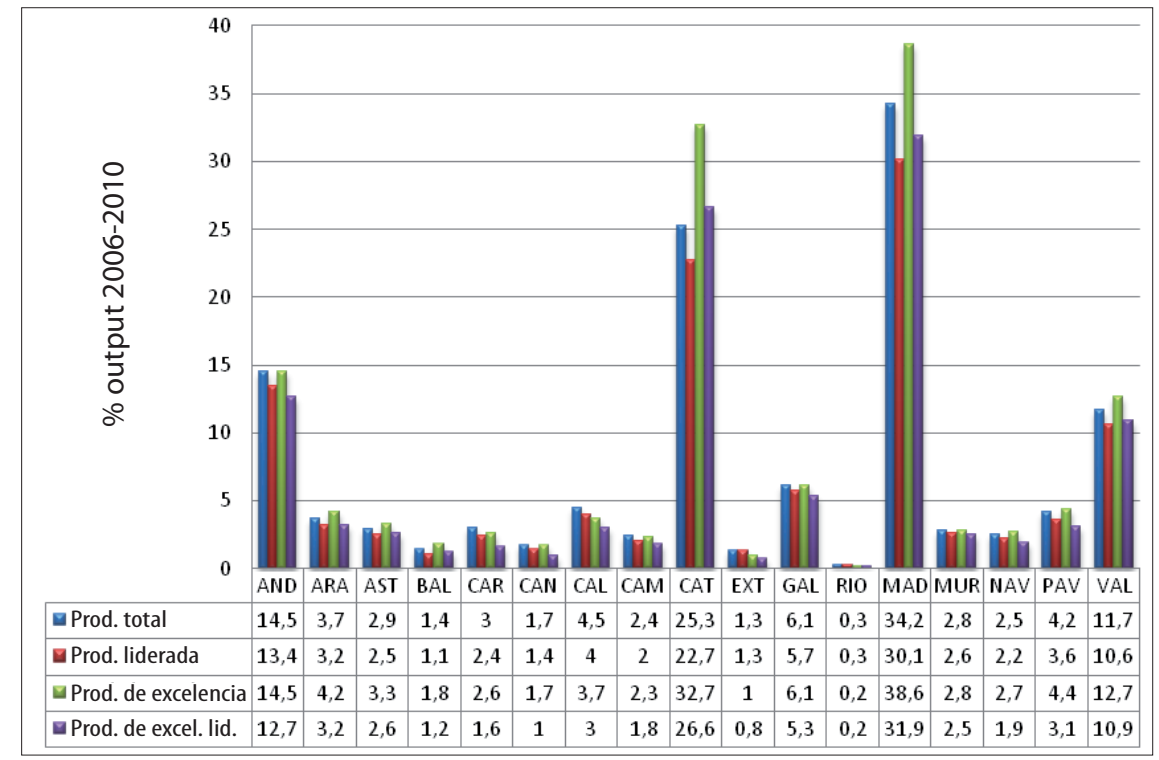

Gráfico 1. Porcentajes de producción total, liderada por españoles, de excelencia (en el 10\% de la producción mundial más citada), y de excelencia liderada de las comunidades autónomas españolas (AND = Andalucía, ARA = Aragón, AST = Asturias, BAL = Baleares, CAR = Cantabria, CAN = Canarias, CAL = Castilla-León, CAM = Castilla-La Mancha, CAT = Catalunya, EXT = Extremadura, GAL = Galicia, RIO = La Rioja, MAD = Madrid, MUR = Murcia, NAV = Navarra, PAV = País Vasco, VAL = Valencia). Período 2006-2010. 
producción de excelencia nacional. De hecho, son las comunidades autónomas que presentan diferencias positivas mas significativas entre su porcentaje de producción de excelencia y bruta (gráfico 1).

Sólo Catalunya tiene un porcentaje de producción de excelencia liderada (27\%) respecto del total nacional que supera a su porcentaje de producción total (25\%)

Ahora bien, si somos capaces de diferenciar el conocimiento científico de excelencia producido con nuestro liderazgo de aquel en el que participamos pero con el liderazgo de otros, estaremos en condiciones de representar mejor nuestras más genuinas capacidades científicas. Tras los reiterados análisis realizados sobre el conjunto de la producción científica española y de otros países y la constatación de que nuestras medias de impacto crecen muy lentamente y nos sitúan en una posición muy postergada en el concierto mundial, debemos enfrentarnos con el análisis de aquella parte de nuestra producción que se encuentra en la zona de excelencia mundial, especialmente cuando en el marco de colaboraciones internacionales nuestros investigadores actúan como actores principales. Desde este punto de vista, en las CCAA se aprecia un sesgo distributivo aún mayor que el observado en la producción científica total. Madrid acumula el mayor porcentaje de excelencia liderada (32\%) del total nacional, pero sólo el de Catalunya (27\%) es superior a su porcentaje de producción total (25\%).

La concentración de producción científica de este nivel es muy destacada en las comunidades de Madrid y Catalunya. Una de las valoraciones que se pueden desprender de estos datos es que se sigue produciendo una concentración de excelencia en el centro científico de España (Catalunya y Madrid) que la transferencia de competencias en materia de investigación no está contribuyendo a paliar y, por tanto, que existe más homogeneidad en la distribución de la producción bruta que en la de la producción de excelencia incluso cuando ésta es liderada por investigadores de nuestro país. En el origen de esta situación está la ubicación en Madrid y Catalunya de los centros de investigación con mayor capacidad de producción científica de excelencia, algunos ejemplos: Centro Nacional de Investigaciones Oncológicas (CNIO), Centro de Regulación Genómica (CRG), Institut Català d'Investigació Química (ICIQ), Instituto de Ciencias Fotónicas (ICFO), Centro Nacional de Biotecnología (CNB), Institut Català d'Oncologia (ICO), Institut Municipal d'Investigació Mèdica (IMIM)...

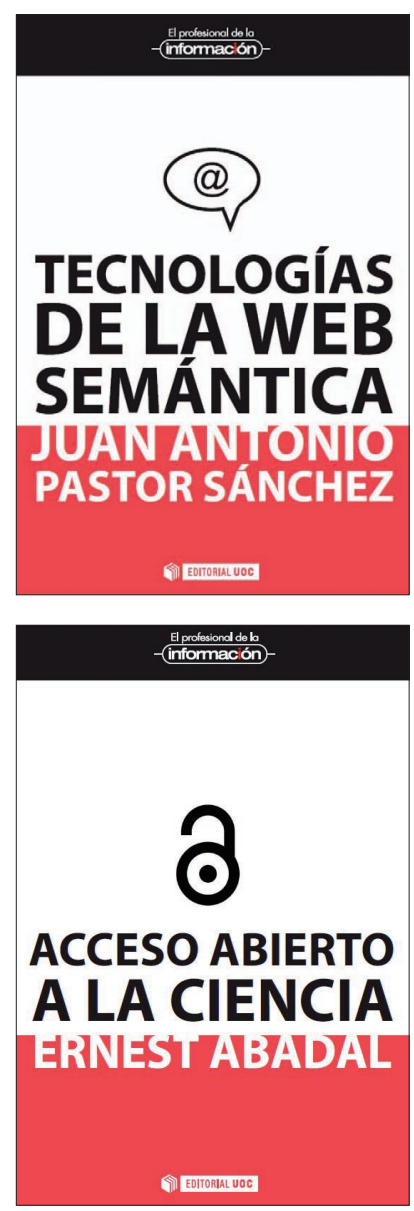

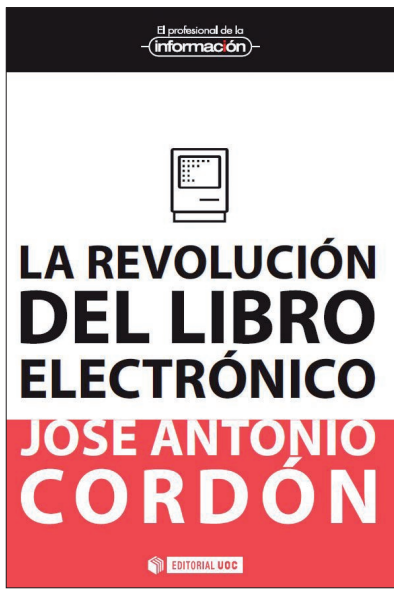

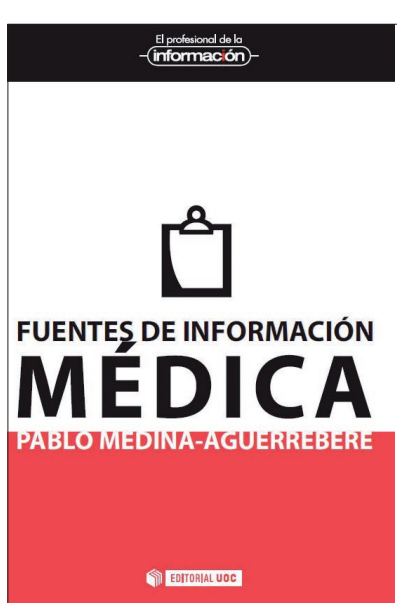

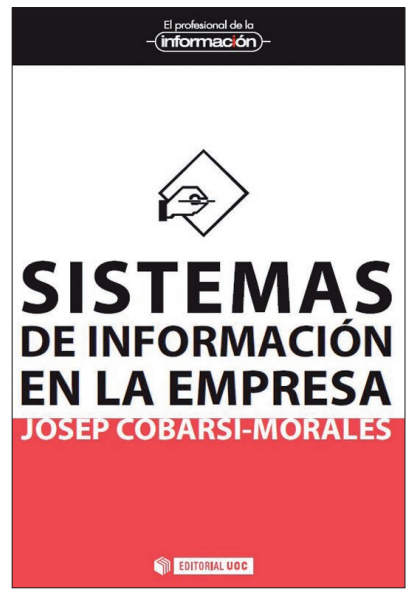

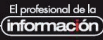

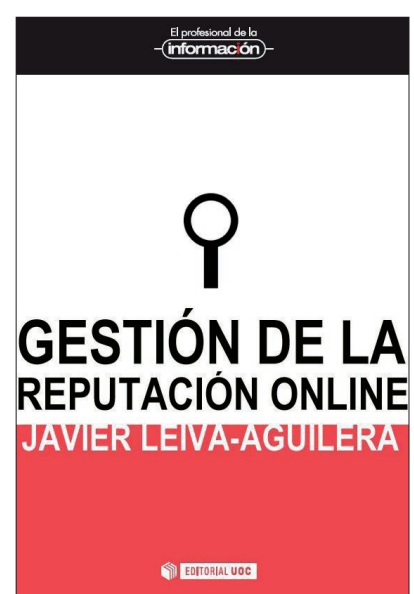

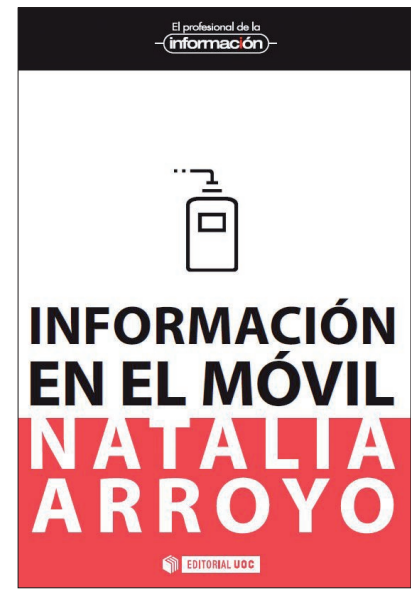

\section{Colección}

de libros de bolsillo EPI-UOC

\section{Más información:}




\section{Creación de Archivos y Bibliotecas Virtuales}

Desde la digitalización de materiales bibliográficos hasta la asignación de metadatos y su implementación en la red, conforme a la normativa internacional.
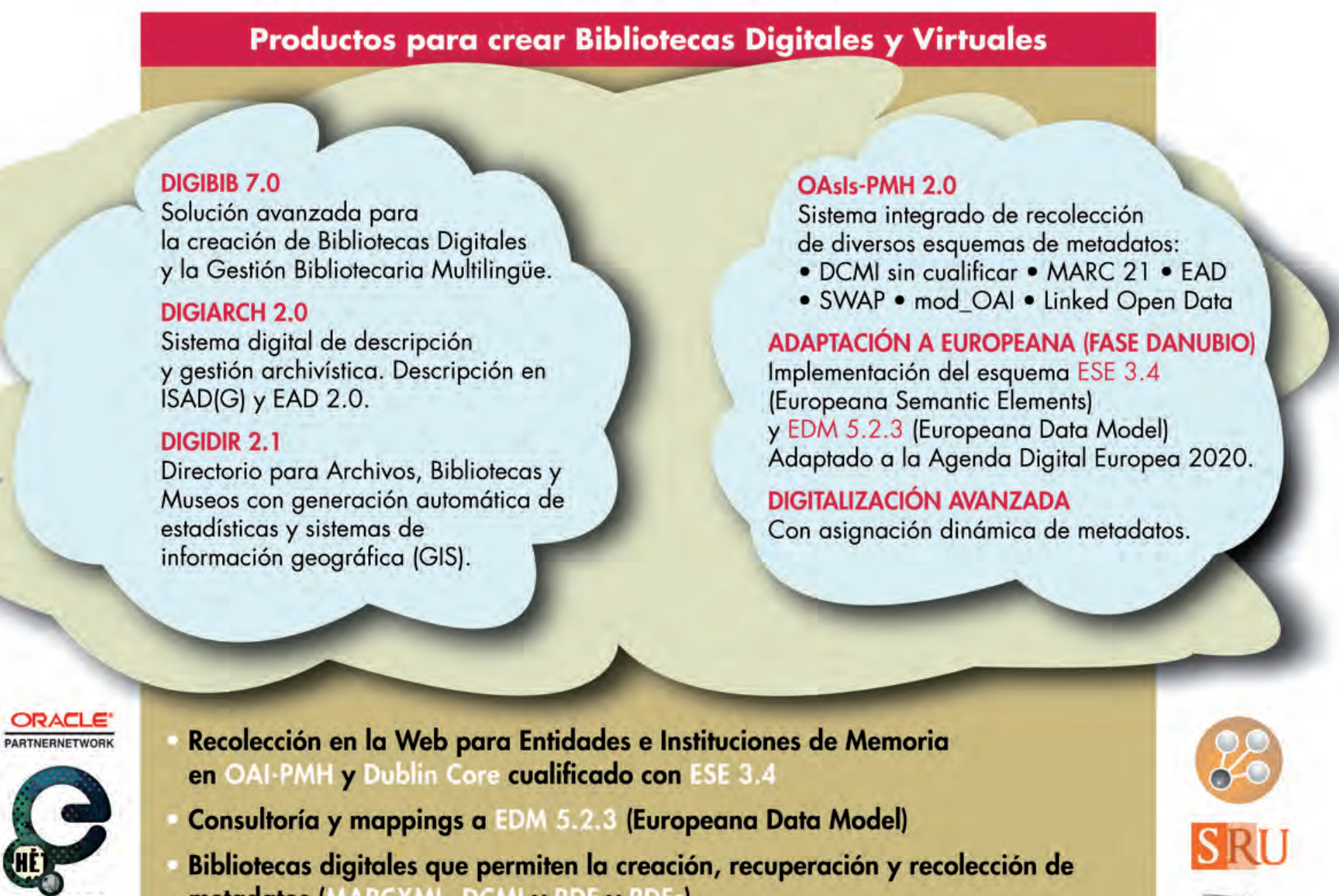

Recolección en la Web para Entidades e Instituciones de Memoria en OAI-PMH y Dublin Core cualificado con ESE 3.4

- Consultoría y mappings a EDM 5.2.3 (Europeana Data Model)

- Bibliotecas digitales que permiten la creación, recuperación y recolección de metadatos (MARCXML, DCMI y RDF y RDFis)

- Archivos Web que facilitan la creación, recuperación y recolección de

W3C metadatos (EAD 2.0 y EAC 2010)

Implementación de la Europeana OpenSearch API

- Adaptación del repositiro OAl para la transmisión de instancios RDF según ORE

Repositorios Institucionales DIGIPRESV para Preservación Digital a largo plazo mediante PREMIS 2.1 y OAIS ISO 14721

Intercambio de metadatos en METS 1.9 (diferentes Profiles) integrando todos los esquemas de metadatos

- Creación de METSRighís para el control de los derechos de autor

- Reconocimiento Óptico de Caracteres OCR y generación dinámica de ALTO (Analyzed Layout and Text Object)

- Generación e integración de registros SKOS mediante MARC 21(Up. 13)/RDA

Creación de eBooks o libros digitales en formatos: ePub y Mobipocket.

Adaptación de DICIBIB a linked Open Data 